\title{
The Sign Language Learning in Deaf Student and Special Education Teacher in Integration Program of Hearing Problem
}

\author{
Mohd Hanafi Mohd Yasin, Mohd Mokhtar Tahar, Safani Bari, Faten Nazren Manaf \\ Universiti Kebangsaan Malaysia, Malaysia \\ Email: mhmy6365@ukm.edu.my
}

\begin{abstract}
This study aims to identify how to learn sign language by deaf students and the level of mastery of sign language by students and teacher of the deaf at a state school in Melaka. In addition, this research would also like to know the major obstacles faced by deaf students and teachers who learn sign language. The instrument that is used in this study is a questionnaire. The respondents contain 50 deaf students and 10 special education teacher of the deaf. Research instrument contains 4 main parts: demographic, the level of mastery of sign language, way to learn sign language and the challenges and obstacles encountered during the study of sign language. Research findings are analyzed using Statistical Package for the Social Science (SPSS) version 22.0 involving the use of descriptive statistics such as frequency, percentage and mean. Research findings indicate that the level of mastery of sign language for problematic pupils hearing and special education teachers is at mid-level. They completely use (KTBM) Kode Tangan Bahasa Melayu (Hand Code of Malay Language) in their daily teaching and communication. In addition, the way to learn sign language for deaf student is through the book while special education teachers also learn it through colleagues. The research also found that challenges as well as the obstacles often encountered to learn sign language for deaf students and special education teachers of the deaf are lack of source reference book of sign language. It is suggested that reference materials such as book and ICT are augmented in all integration program and special education of the deaf.
\end{abstract}

Keywords: Kode Tangan Bahasa Melayu, sign language, the medium of instruction, challenge

Language is a mean of connector and ways to interact among other. The approach to the use of sign language in the deaf students is the real use of sign language in the learning and teaching of languages for these groups (Paul \& Quigley 1994). The use of sign language allows them to interact and communicate with the community. In Malaysia, there is a (BIM) Bahasa Isyarat Malaysia (Malaysian sign language) and (KTBM) Kode Tangan Bahasa Melayu (Hand Code of MalayLanguage) are often used. According to Lim, 2006, BIM is major sign language for the students with hearing barriers. BIM is also the communication language used in their daily life. BIM has become an identity for this group was in Malaysia because the sign form used is according to their way of life without getting influence than other elements (Sazali Shaari, 2004). KTBM anyway, is a code that is delivered through style of hand signal that represents each vocabulary in Malay (Ministry of education of Malaysia, 1985). Hand code used needs to meet ethical vocabulary of Malay language such as code for basic words, the plural words, multiple words, affixes and others.

The combination of use of BIM and KTBM often confuse students and teachers when having communication. It happens because BIM is not thought at formal school. But almost all deaf students communicate with BIM (Goh \& Teh, 1993). They stated that it was easier to understand BIM than KTBM. They consider the BIM is main language of the deaf community in Malaysia (Lim, 2006). As like BIM, KTBM also focuses on hand signals and the principle of the correct Malay grammar.

The lack of the use of electronic media in learning sign language affects its use by deaf students. Electronic media and ICT could interest students in learning because they have the addition of music and visual form that can simplify their understanding (Plamen \& Rodgers, 2003). But there are also study that states that teachers whoare less skilled in ICT becomes a barrier because of the lack of training and practice.

Study has shown that individual with problems of hearing since birth will face constrains and difficulties in the learning process. This is due to their poor language proficiency. It causes them difficult to communicate. This issue gives the negative impact such as slow language development. The environmental condition such as the area of living and learning environment that is not conducive also add the delay in their language development. By all statements above, this research is conducted to identiy effective way of learning sign language in the learning process and mastering sign language for deaf students and special education teachers at school. 
Table 1. Demografi murid

\begin{tabular}{lcc}
\hline Item & Kekerapan & Peratus \\
\hline Jantina & 27 & \\
Lelaki & 23 & 54.0 \\
Perempuan & & 46.0 \\
Bangsa & 38 & \\
Melayu & 8 & 76.0 \\
Cina & 4 & 16.0 \\
India & 0 & 8.0 \\
Lain-lain & & 0 \\
Umur & 8 & \\
13-14 tahun & 30 & 16.0 \\
15-16 tahun & 12 & 60.0 \\
17 tahun keats & & 24.0 \\
Penguasaan Bahasa Isyarat & 13 & \\
Baik & 35 & 26.0 \\
Sederhana & 2 & 70.0 \\
Lemah & & 4.0 \\
Bahasa isyarat Dominan & 46 & 92.0 \\
KTBM & 4 & 8.0 \\
BIM & & \\
\hline & & \\
& &
\end{tabular}

Table 2. Demografi Guru

\begin{tabular}{lcc}
\hline Item & Kekerapan & Peratus \\
\hline Jantina & 1 & \\
Lelaki & 9 & 10.0 \\
Perempuan & & 90.0 \\
Bangsa & 7 & \\
Melayu & 2 & 70.0 \\
Cina & 0 & 20.0 \\
India & 1 & 0 \\
Lain-lain & & 10.0 \\
Tahap pendidikan & 1 & \\
Diploma P.Khas & 6 & 10.0 \\
Ijazah P.Khas & 3 & 60.0 \\
Lain-lain & & 30.0 \\
Tempoh berkhidmat & 1 & \\
1-5 tahun & 6 & 10.0 \\
6-10 tahun & 1 & 60.0 \\
10-15 tahun & 2 & 10.0 \\
15 tahun keatas & & 20.0 \\
Bahasa isyarat Dominan & 10 & 10.0 \\
KTBM & 0 & 0 \\
BIM & & \\
\hline & & \\
& &
\end{tabular}

Secara keseluruhannya, analisis deskriptif bagi item-item soal selidik dapat diterangkan melalui jadual-jadual berikut :

Table 3. Tahap bahasa isyarat murid bermasalah pendengaran

\begin{tabular}{|c|c|c|c|c|c|c|c|c|}
\hline BIL & PENYATAAN & $\begin{array}{c}\text { STS } \\
\mathbf{f} \\
\%\end{array}$ & $\begin{array}{c}\text { TS } \\
\text { f } \\
\%\end{array}$ & $\begin{array}{c}\text { TP } \\
\mathbf{f} \\
\%\end{array}$ & $\begin{array}{c}\mathbf{S} \\
\mathbf{f} \\
\%\end{array}$ & $\begin{array}{c}\text { SS } \\
\text { f } \\
\%\end{array}$ & MIN & Tahap \\
\hline 1. & $\begin{array}{l}\text { Saya mampu untuk menguasai bahasa } \\
\text { isyarat dengan baik. }\end{array}$ & $\begin{array}{c}3 \\
6.0\end{array}$ & $\begin{array}{c}3 \\
6.0\end{array}$ & $\begin{array}{c}10 \\
20.0\end{array}$ & $\begin{array}{c}32 \\
64.0\end{array}$ & $\begin{array}{c}2 \\
4.0\end{array}$ & 3.54 & Sederhana \\
\hline 2. & $\begin{array}{l}\text { Saya selalu berkomunikasi menggunakan } \\
\text { bahasa isyarat yang betul. }\end{array}$ & $\begin{array}{c}3 \\
6.0\end{array}$ & $\begin{array}{c}2 \\
4.0\end{array}$ & $\begin{array}{c}16 \\
32.0\end{array}$ & $\begin{array}{c}25 \\
50.0\end{array}$ & $\begin{array}{c}4 \\
8.0\end{array}$ & 3.50 & Sederhana \\
\hline 3. & $\begin{array}{l}\text { BIM merupakan bahasa komunikasi utama } \\
\text { saya. }\end{array}$ & $\begin{array}{c}3 \\
6.0\end{array}$ & $\begin{array}{c}8 \\
16.0\end{array}$ & $\begin{array}{c}26 \\
52.0\end{array}$ & $\begin{array}{c}12 \\
24.0\end{array}$ & $\begin{array}{c}1 \\
2.0\end{array}$ & 3.00 & Sederhana \\
\hline 4. & $\begin{array}{l}\text { KTBM merupakan bahasa komunikasi } \\
\text { utama saya. }\end{array}$ & 0 & $\begin{array}{c}4 \\
8.0\end{array}$ & $\begin{array}{c}28 \\
56.0\end{array}$ & $\begin{array}{c}15 \\
30.0\end{array}$ & $\begin{array}{c}3 \\
6.0\end{array}$ & 3.35 & Sederhana \\
\hline 5. & BIM mudah untuk difahami. & $\begin{array}{c}2 \\
4.0\end{array}$ & $\begin{array}{c}4 \\
8.0\end{array}$ & $\begin{array}{c}29 \\
58.0\end{array}$ & $\begin{array}{c}13 \\
26.0\end{array}$ & $\begin{array}{c}2 \\
4.0\end{array}$ & 3.18 & Sederhana \\
\hline 6. & KTBM lebih mudah untuk dikuasai. & $\begin{array}{c}3 \\
6.0\end{array}$ & $\begin{array}{c}2 \\
4.0\end{array}$ & $\begin{array}{c}27 \\
54.0\end{array}$ & $\begin{array}{c}16 \\
32.0\end{array}$ & $\begin{array}{c}2 \\
4.0\end{array}$ & 3.24 & Sederhana \\
\hline 7. & $\begin{array}{l}\text { Saya dapat membezakan ayat menggunakan } \\
\text { BIM dan KTBM }\end{array}$ & $\begin{array}{c}3 \\
6.0\end{array}$ & $\begin{array}{c}3 \\
6.0\end{array}$ & $\begin{array}{c}20 \\
40.0\end{array}$ & $\begin{array}{c}18 \\
36.0\end{array}$ & $\begin{array}{c}6 \\
12.0\end{array}$ & 3.42 & Sederhana \\
\hline 8. & $\begin{array}{l}\text { Saya menggunakan KTBM bagi proses } \\
\text { pembelajaran di dalam kelas. }\end{array}$ & $\begin{array}{c}3 \\
6.0\end{array}$ & $\begin{array}{c}4 \\
8.0\end{array}$ & $\begin{array}{c}12 \\
24.0\end{array}$ & $\begin{array}{c}16 \\
32.0\end{array}$ & $\begin{array}{c}15 \\
30.0\end{array}$ & 3.72 & Tinggi \\
\hline 9. & $\begin{array}{l}\text { Terdapat banyak perbezaan diantara KTBM } \\
\text { dan BIM. }\end{array}$ & $\begin{array}{c}2 \\
4.0\end{array}$ & $\begin{array}{c}3 \\
6.0\end{array}$ & $\begin{array}{c}32 \\
64.0\end{array}$ & $\begin{array}{c}9 \\
18.0\end{array}$ & $\begin{array}{c}4 \\
8.0\end{array}$ & 3.20 & Sederhana \\
\hline 10. & $\begin{array}{l}\text { BIM mempunyai susunan ayat yang tidak } \\
\text { mematuhi tatabahasa bahasa Melayu. }\end{array}$ & $\begin{array}{c}1 \\
2.0\end{array}$ & $\begin{array}{c}8 \\
16.0\end{array}$ & $\begin{array}{c}28 \\
56.0\end{array}$ & $\begin{array}{c}11 \\
22.0\end{array}$ & $\begin{array}{c}2 \\
4.0\end{array}$ & 3.10 & Sederhana \\
\hline 11. & $\begin{array}{l}\text { KTBM menepati ciri-ciri tatabahasa bahasa } \\
\text { Melayu. }\end{array}$ & $\begin{array}{c}2 \\
4.0\end{array}$ & $\begin{array}{c}3 \\
6.0\end{array}$ & $\begin{array}{c}30 \\
60.0\end{array}$ & $\begin{array}{c}14 \\
28.0\end{array}$ & $\begin{array}{c}1 \\
2.0\end{array}$ & 3.18 & Sederhana \\
\hline 12. & $\begin{array}{l}\text { Saya boleh mengisyaratkan BIM dengan } \\
\text { betul }\end{array}$ & $\begin{array}{c}4 \\
8.0\end{array}$ & $\begin{array}{c}2 \\
4.0\end{array}$ & $\begin{array}{c}27 \\
54.0\end{array}$ & $\begin{array}{c}15 \\
30.0\end{array}$ & $\begin{array}{c}2 \\
4.0\end{array}$ & 3.18 & Sederhana \\
\hline 13. & $\begin{array}{l}\text { Saya boleh mengisyaratkan KTBM dengan } \\
\text { betul }\end{array}$ & $\begin{array}{c}2 \\
4.0\end{array}$ & $\begin{array}{c}3 \\
6.0\end{array}$ & $\begin{array}{c}19 \\
38.0\end{array}$ & $\begin{array}{c}20 \\
40.0\end{array}$ & $\begin{array}{c}6 \\
12.0\end{array}$ & 3.50 & Sederhana \\
\hline 14. & BIM lebih mudah untuk dipelajari. & $\begin{array}{c}4 \\
8.0\end{array}$ & $\begin{array}{c}2 \\
4.0\end{array}$ & $\begin{array}{c}23 \\
46.0\end{array}$ & $\begin{array}{c}17 \\
34.0\end{array}$ & $\begin{array}{c}4 \\
8.0\end{array}$ & 3.30 & Sederhana \\
\hline 15. & $\begin{array}{l}\text { Pembelajaran menggunakan KTBM } \\
\text { membantu perkembangan bahasa saya. }\end{array}$ & $\begin{array}{c}3 \\
6.0\end{array}$ & $\begin{array}{c}3 \\
6.0\end{array}$ & $\begin{array}{c}6 \\
12.0\end{array}$ & $\begin{array}{c}13 \\
26.0\end{array}$ & $\begin{array}{c}25 \\
50.0\end{array}$ & 4.08 & Tinggi \\
\hline & Jumlah purata min keseluruhan & & & & & & 3.15 & Sederhana \\
\hline
\end{tabular}


Table 4. Tahap bahasa isyarat guru pendidikan khas bermasalah pendengaran

\begin{tabular}{|c|c|c|c|c|c|c|c|c|}
\hline BIL & PENYATAAN & $\begin{array}{l}\text { STS } \\
\text { f } \\
\%\end{array}$ & $\begin{array}{c}\text { TS } \\
\text { f } \\
\%\end{array}$ & $\begin{array}{c}\text { TP } \\
\text { f } \\
\%\end{array}$ & $\begin{array}{l}\mathrm{S} \\
\mathbf{f} \\
\%\end{array}$ & $\begin{array}{c}\text { SS } \\
\mathbf{f} \\
\%\end{array}$ & MIN & Markah \\
\hline 1. & $\begin{array}{l}\text { Saya mampu untuk menguasai bahasa } \\
\text { isyarat dengan baik. }\end{array}$ & 0 & 0 & $\begin{array}{c}1 \\
10.0\end{array}$ & $\begin{array}{c}8 \\
80.0\end{array}$ & $\begin{array}{c}1 \\
10.0\end{array}$ & 4.00 & Tinggi \\
\hline 2. & $\begin{array}{l}\text { Saya selalu berkomunikasi menggunakan } \\
\text { bahasa isyarat yang betul. }\end{array}$ & 0 & 0 & $\begin{array}{c}3 \\
30.0\end{array}$ & $\begin{array}{c}6 \\
60.0\end{array}$ & $\begin{array}{c}1 \\
10.0\end{array}$ & 3.80 & Tinggi \\
\hline 3. & $\begin{array}{l}\text { BIM merupakan bahasa komunikasi utama } \\
\text { saya. }\end{array}$ & 0 & $\begin{array}{c}5 \\
50.0\end{array}$ & $\begin{array}{c}5 \\
50.0\end{array}$ & 0 & 0 & 2.50 & Sederhana \\
\hline 4. & $\begin{array}{l}\text { KTBM merupakan bahasa komunikasi } \\
\text { utama saya. }\end{array}$ & 0 & 0 & $\begin{array}{c}2 \\
20.0\end{array}$ & $\begin{array}{c}6 \\
60.0\end{array}$ & $\begin{array}{c}2 \\
20.0\end{array}$ & 4.00 & Tinggi \\
\hline 5. & BIM mudah untuk difahami. & 0 & $\begin{array}{c}2 \\
20.0\end{array}$ & $\begin{array}{c}7 \\
70.0\end{array}$ & $\begin{array}{c}1 \\
10.0\end{array}$ & 0 & 2.90 & Sederhana \\
\hline 6. & KTBM lebih mudah untuk dikuasai. & 0 & 0 & $\begin{array}{c}2 \\
20.0\end{array}$ & $\begin{array}{c}8 \\
80.0\end{array}$ & 0 & 3.80 & Tinggi \\
\hline 7. & $\begin{array}{l}\text { Saya dapat membezakan ayat menggunakan } \\
\text { BIM dan KTBM }\end{array}$ & 0 & 0 & $\begin{array}{c}5 \\
50.0\end{array}$ & $\begin{array}{c}2 \\
20.0\end{array}$ & $\begin{array}{c}3 \\
30.0\end{array}$ & 3.80 & Tinggi \\
\hline 8. & $\begin{array}{l}\text { Saya mengutamakan penggunaan KTBM } \\
\text { dalam proses pengajaran dan pembelajaran. }\end{array}$ & 0 & 0 & $\begin{array}{c}1 \\
10.0\end{array}$ & $\begin{array}{c}5 \\
50.0\end{array}$ & $\begin{array}{c}4 \\
40.0\end{array}$ & 4.30 & Tinggi \\
\hline 9. & $\begin{array}{l}\text { Terdapat banyak perbezaan diantara KTBM } \\
\text { dan BIM. }\end{array}$ & 0 & 0 & $\begin{array}{c}4 \\
40.0\end{array}$ & $\begin{array}{c}3 \\
30.0\end{array}$ & $\begin{array}{c}3 \\
30.0\end{array}$ & 3.90 & Tinggi \\
\hline 10. & $\begin{array}{l}\text { BIM mempunyai susunan ayat yang tidak } \\
\text { mematuhi tatabahasa bahasa Melayu. }\end{array}$ & 0 & 0 & $\begin{array}{c}5 \\
50.0\end{array}$ & $\begin{array}{c}4 \\
40.0\end{array}$ & $\begin{array}{c}1 \\
10.0\end{array}$ & 3.60 & Sederhana \\
\hline 11. & $\begin{array}{l}\text { KTBM menepati ciri-ciri tatabahasa bahasa } \\
\text { Melayu. }\end{array}$ & 0 & 0 & $\begin{array}{c}3 \\
30.0\end{array}$ & $\begin{array}{c}3 \\
30.0\end{array}$ & $\begin{array}{c}4 \\
40.0\end{array}$ & 4.10 & Tinggi \\
\hline 12. & $\begin{array}{l}\text { Saya boleh mengisyaratkan BIM dengan } \\
\text { betul }\end{array}$ & 0 & $\begin{array}{c}1 \\
10.0\end{array}$ & $\begin{array}{c}7 \\
70.0\end{array}$ & $\begin{array}{c}2 \\
20.0\end{array}$ & 0 & 3.10 & Sederhana \\
\hline 13. & $\begin{array}{l}\text { Saya boleh mengisyaratkan KTBM dengan } \\
\text { betul }\end{array}$ & 0 & 0 & $\begin{array}{c}2 \\
20.0\end{array}$ & $\begin{array}{c}3 \\
30.0\end{array}$ & $\begin{array}{c}5 \\
50.0\end{array}$ & 4.30 & Tinggi \\
\hline 14. & BIM lebih mudah untuk dipelajari. & 0 & $\begin{array}{c}3 \\
30.0\end{array}$ & $\begin{array}{c}7 \\
70.0\end{array}$ & 0 & 0 & 2.70 & Sederhana \\
\hline 15. & $\begin{array}{l}\text { Pengajaran menggunakan KTBM } \\
\text { membantu perkembangan bahasa murid- } \\
\text { murid bermasalah pendengaran }\end{array}$ & 0 & 0 & 0 & $\begin{array}{c}5 \\
50.0\end{array}$ & $\begin{array}{c}5 \\
50.0\end{array}$ & 4.50 & Tinggi \\
\hline
\end{tabular}

Table 5. medium pembelajaran bahasa isyarat murid bermasalah pendengaran

\begin{tabular}{|c|c|c|c|c|c|c|c|c|}
\hline BIL & PENYATAAN & $\begin{array}{c}\text { STS } \\
\text { f } \\
\%\end{array}$ & $\begin{array}{c}\text { TS } \\
\mathbf{f} \\
\%\end{array}$ & $\begin{array}{c}\text { TP } \\
\mathbf{f} \\
\%\end{array}$ & $\begin{array}{l}\mathbf{S} \\
\mathbf{f} \\
\%\end{array}$ & $\begin{array}{c}\text { SS } \\
\text { f } \\
\%\end{array}$ & MIN & Markah \\
\hline 1. & Saya belajar bahasa isyarat melalui buku. & $\begin{array}{c}4 \\
8.0\end{array}$ & $\begin{array}{c}3 \\
6.0\end{array}$ & $\begin{array}{c}4 \\
8.0\end{array}$ & $\begin{array}{c}36 \\
72.0\end{array}$ & $\begin{array}{c}3 \\
6.0\end{array}$ & 3.62 & Sederhana \\
\hline 2. & $\begin{array}{l}\text { Saya belajar bahasa isyarat melalui rakan } \\
\text { sebaya. }\end{array}$ & $\begin{array}{c}1 \\
2.0\end{array}$ & $\begin{array}{c}3 \\
6.0\end{array}$ & $\begin{array}{c}12 \\
24.0\end{array}$ & $\begin{array}{c}33 \\
66.0\end{array}$ & $\begin{array}{c}1 \\
2.0\end{array}$ & 3.60 & Sederhana \\
\hline 3. & Saya belajar bahasa isyarat melalui web. & $\begin{array}{c}4 \\
8.0\end{array}$ & $\begin{array}{c}11 \\
22.0\end{array}$ & $\begin{array}{c}29 \\
58.0\end{array}$ & $\begin{array}{c}6 \\
12.0\end{array}$ & 0 & 2.74 & Sederhana \\
\hline 4. & $\begin{array}{l}\text { Medium penggunaan web menjadi medium } \\
\text { pembelajaran yang utama saya. }\end{array}$ & $\begin{array}{c}7 \\
14.0\end{array}$ & $\begin{array}{c}8 \\
16.0\end{array}$ & $\begin{array}{c}26 \\
52.0\end{array}$ & $\begin{array}{c}7 \\
14.0\end{array}$ & $\begin{array}{c}2 \\
4.0\end{array}$ & 2.78 & Sederhana \\
\hline 5. & $\begin{array}{l}\text { Belajar bahasa isyarat melalui buku lebih } \\
\text { mudah untuk difahami. }\end{array}$ & $\begin{array}{c}1 \\
2.0\end{array}$ & $\begin{array}{c}2 \\
4.0\end{array}$ & $\begin{array}{c}9 \\
18.0\end{array}$ & $\begin{array}{c}26 \\
52.0\end{array}$ & $\begin{array}{c}12 \\
24.0\end{array}$ & 3.92 & Tinggi \\
\hline 6. & $\begin{array}{l}\text { Pembelajaran bahasa isyarat melalui laman } \\
\text { web memudahkan pemahaman saya. }\end{array}$ & 0 & $\begin{array}{c}10 \\
20.0\end{array}$ & $\begin{array}{c}26 \\
52.0\end{array}$ & $\begin{array}{c}12 \\
24.0\end{array}$ & $\begin{array}{c}2 \\
4.0\end{array}$ & 3.12 & Sederhana \\
\hline 7. & $\begin{array}{l}\text { Proses komunikasi dengan rakan sebaya } \\
\text { serta guru membantu dalam penguasaan }\end{array}$ & $\begin{array}{c}3 \\
6.0\end{array}$ & $\begin{array}{c}4 \\
8.0\end{array}$ & $\begin{array}{c}6 \\
12.0\end{array}$ & $\begin{array}{c}28 \\
56.0\end{array}$ & $\begin{array}{c}9 \\
18.0\end{array}$ & 3.72 & Tinggi \\
\hline
\end{tabular}


8. Buku merupakan medium rujukan saya di dalam bilik darjah.

9. Saya dapat menambah perkataan bahasa isyarat yang baru melalui interaksi dengan rakan sebaya

10. Pembelajaran melalui tayangan video memudahkan proses pembelajaran bahasa isyarat.

11. Video suara dan gaya isyarat tangan membantu pembelajaran bahasa isyarat.

12. Buku merupakan sumber yang mudah untuk diakses dan mudah untuk dibawa.

13. Aplikasi web bahasa isyarat yang boleh diakses dengan mudah mampu untuk menggantikan buku. pembelajaran bahasa isyarat.

\begin{tabular}{ccccccc}
5 & 3 & 13 & 22 & 7 & 3.46 & Sederhana \\
10.0 & 6.0 & 26.0 & 44.0 & 14.0 & & \\
3 & 1 & 17 & 22 & 7 & 3.58 & Sederhana \\
6.0 & 2.0 & 34.0 & 44.0 & 14.0 & & \\
& & & & & & \\
1 & 5 & 18 & 24 & 2 & 3.42 & Sederhana \\
2.0 & 10.0 & 36.0 & 48.0 & 4.0 & & \\
& & & & & & \\
1 & 5 & 18 & 17 & 9 & 3.56 & Sederhana \\
2.0 & 10.0 & 36.0 & 34.0 & 18.0 & & \\
3 & 4 & 12 & 24 & 7 & 3.56 & Sederhana \\
6.0 & 8.0 & 24.0 & 48.0 & 14.0 & & \\
4 & 8 & 23 & 13 & 2 & 3.02 & Sederhana \\
8.0 & 16.0 & 46.0 & 26.0 & 4.0 & & \\
& & & & & & \\
0 & 3 & 9 & 33 & 5 & 3.80 & Tinggi \\
& 6.0 & 18.0 & 66.0 & 10.0 & & \\
2 & 4 & 16 & 24 & 4 & 3.48 & Sederhana \\
4.0 & 8.0 & 32.0 & 48.0 & 8.0 & & \\
\hline & & & & & $\mathbf{3 . 4 3}$ & Sederhana
\end{tabular}

Table 6. medium pembelajaran bahasa isyarat guru bermasalah pendengaran

\begin{tabular}{|c|c|c|c|c|c|c|c|c|}
\hline BIL & PENYATAAN & $\begin{array}{l}\text { STS } \\
\text { f } \\
\%\end{array}$ & $\begin{array}{c}\text { TS } \\
\mathbf{f} \\
\%\end{array}$ & $\begin{array}{c}\text { TP } \\
\mathbf{f} \\
\%\end{array}$ & $\begin{array}{c}\mathrm{S} \\
\mathbf{f} \\
\%\end{array}$ & $\begin{array}{c}\text { SS } \\
\mathbf{f} \\
\%\end{array}$ & MIN & Markah \\
\hline 1. & Saya belajar bahasa isyarat melalui buku. & 0 & 0 & 2 & $\begin{array}{c}6 \\
60.0\end{array}$ & $\begin{array}{c}2 \\
20.0\end{array}$ & 4.00 & Tinggi \\
\hline 2. & $\begin{array}{l}\text { Saya belajar bahasa isyarat melalui rakan } \\
\text { sekerja. }\end{array}$ & 0 & 0 & 0 & $\begin{array}{c}8 \\
80.0\end{array}$ & $\begin{array}{c}2 \\
20.0\end{array}$ & 4.20 & Tinggi \\
\hline 3. & Saya belajar bahasa isyarat melalui web. & 0 & $\begin{array}{c}2 \\
20.0\end{array}$ & $\begin{array}{c}6 \\
60.0\end{array}$ & $\begin{array}{c}1 \\
10.0\end{array}$ & $\begin{array}{c}1 \\
10.0\end{array}$ & 3.10 & sederhana \\
\hline 4. & $\begin{array}{l}\text { Saya mempelajari bahasa isyarat hanya } \\
\text { melalui rakan sekerja. }\end{array}$ & 0 & $\begin{array}{c}3 \\
30.0\end{array}$ & $\begin{array}{c}3 \\
30.0\end{array}$ & $\begin{array}{c}3 \\
30.0\end{array}$ & $\begin{array}{c}1 \\
10.0\end{array}$ & 3.20 & Sederhana \\
\hline 5. & $\begin{array}{l}\text { Medium penggunaan web menjadi medium } \\
\text { pembelajaran yang utama saya. }\end{array}$ & $\begin{array}{c}1 \\
10.0\end{array}$ & $\begin{array}{c}3 \\
30.0\end{array}$ & $\begin{array}{c}4 \\
40.0\end{array}$ & $\begin{array}{c}2 \\
20.0\end{array}$ & 0 & 2.70 & Sederhana \\
\hline 6. & $\begin{array}{l}\text { Belajar bahasa isyarat melalui buku lebih } \\
\text { mudah untuk difahami. }\end{array}$ & 0 & $\begin{array}{c}3 \\
30.0\end{array}$ & $\begin{array}{c}2 \\
20.0\end{array}$ & $\begin{array}{c}3 \\
30.0\end{array}$ & $\begin{array}{c}3 \\
30.0\end{array}$ & 3.60 & Sederhana \\
\hline 7. & $\begin{array}{l}\text { Pembelajaran bahasa isyarat melalui laman } \\
\text { web membantu saya. }\end{array}$ & 0 & $\begin{array}{c}2 \\
20.0\end{array}$ & $\begin{array}{c}3 \\
30.0\end{array}$ & $\begin{array}{c}4 \\
40.0\end{array}$ & $\begin{array}{c}1 \\
10.0\end{array}$ & 3.40 & Sederhana \\
\hline 8. & $\begin{array}{l}\text { Proses komunikasi dengan rakan sekerja } \\
\text { serta pelajar sangat membantu dalam } \\
\text { penguasaan bahasa isyarat saya. }\end{array}$ & 0 & 0 & $\begin{array}{c}1 \\
10.0\end{array}$ & $\begin{array}{c}3 \\
30.0\end{array}$ & $\begin{array}{c}6 \\
60.0\end{array}$ & 4.50 & Tinggi \\
\hline 9. & $\begin{array}{l}\text { Buku merupakan medium rujukan saya } \\
\text { yang utama setiap kali berkomunikasi } \\
\text { dengan pelajar. }\end{array}$ & 0 & $\begin{array}{c}1 \\
10.0\end{array}$ & $\begin{array}{c}2 \\
20.0\end{array}$ & $\begin{array}{c}1 \\
10.0\end{array}$ & 60.0 & 4.20 & Tinggi \\
\hline 10. & $\begin{array}{l}\text { Interaksi dengan rakan dapat menambahkan } \\
\text { perkataan baru bahasa isyarat saya. }\end{array}$ & 0 & 0 & 0 & $\begin{array}{c}5 \\
50.0\end{array}$ & $\begin{array}{c}5 \\
50.0\end{array}$ & 4.50 & Tinggi \\
\hline 11. & $\begin{array}{l}\text { Pembelajaran melalui tayangan video } \\
\text { memudahkan proses pembelajaran bahasa } \\
\text { isyarat. }\end{array}$ & 0 & 0 & $\begin{array}{c}3 \\
30.0\end{array}$ & $\begin{array}{c}5 \\
50.0\end{array}$ & $\begin{array}{c}2 \\
20.0\end{array}$ & 3.90 & Tinggi \\
\hline 12. & $\begin{array}{l}\text { Buku merupakan medium pembelajaran } \\
\text { visual yang baik. }\end{array}$ & 0 & 0 & $\begin{array}{c}4 \\
40.0\end{array}$ & $\begin{array}{c}4 \\
40.0\end{array}$ & $\begin{array}{c}2 \\
20.0\end{array}$ & 3.80 & Tinggi \\
\hline 13. & $\begin{array}{l}\text { Video beserta suara dan gaya isyarat tangan } \\
\text { sangat membantu pembelajaran bahasa } \\
\text { isyarat. }\end{array}$ & 0 & 0 & 0 & $\begin{array}{c}7 \\
70.0\end{array}$ & $\begin{array}{c}3 \\
30.0\end{array}$ & 4.30 & Tinggi \\
\hline 14. & $\begin{array}{l}\text { Buku merupakan sumber yang mudah untuk } \\
\text { diakses dan mudah untuk dibawa. }\end{array}$ & 0 & 0 & $\begin{array}{c}1 \\
10.0\end{array}$ & $\begin{array}{c}8 \\
80.0\end{array}$ & $\begin{array}{c}1 \\
10.0\end{array}$ & 4.00 & Tinggi \\
\hline
\end{tabular}


15. Aplikasi laman web bahasa isyarat yang boleh diakses dengan mudah mampu untuk 0 menggantikan buku.

Table 7. Halangan dan Cabaran Murid Bermasalah Pendengaran dalam Mempelajari Bahasa Isyarat

\begin{tabular}{|c|c|c|c|c|c|c|c|c|}
\hline BIL & PENYATAAN & $\begin{array}{c}\text { STS } \\
\text { f } \\
\% \\
\end{array}$ & $\begin{array}{c}\text { TS } \\
\text { f } \\
\%\end{array}$ & $\begin{array}{c}\text { TP } \\
\text { f } \\
\%\end{array}$ & $\begin{array}{c}S \\
\text { f } \\
\%\end{array}$ & $\begin{array}{c}\text { SS } \\
\text { f } \\
\%\end{array}$ & MIN & Markah \\
\hline 1. & $\begin{array}{l}\text { Saya sukar mempelajari bahasa isyarat } \\
\text { kerana kekurangan kemudahan ICT. }\end{array}$ & $\begin{array}{c}7 \\
14.0\end{array}$ & $\begin{array}{c}4 \\
8.0\end{array}$ & $\begin{array}{c}12 \\
24.0\end{array}$ & $\begin{array}{c}26 \\
52.0\end{array}$ & $\begin{array}{c}1 \\
2.0\end{array}$ & 3.20 & Sederhana \\
\hline 2. & $\begin{array}{l}\text { KTBM mengambil masa yang lama untuk } \\
\text { dipelajari dan dihafal }\end{array}$ & $\begin{array}{c}3 \\
6.0\end{array}$ & $\begin{array}{c}3 \\
6.0\end{array}$ & $\begin{array}{c}27 \\
54.0\end{array}$ & $\begin{array}{c}13 \\
26.0\end{array}$ & $\begin{array}{c}4 \\
8.0\end{array}$ & 3.24 & Sederhana \\
\hline 3. & Saya malas untuk belajar bahasa isyarat & $\begin{array}{c}5 \\
10.0\end{array}$ & $\begin{array}{c}34 \\
68.0\end{array}$ & $\begin{array}{c}8 \\
16.0\end{array}$ & $\begin{array}{c}2 \\
4.0\end{array}$ & $\begin{array}{c}1 \\
2.0\end{array}$ & 2.20 & Rendah \\
\hline 4. & $\begin{array}{l}\text { Saya mengalami kecelaruan dan kekeliruan } \\
\text { antara BIM dan KTBM. }\end{array}$ & $\begin{array}{c}1 \\
2.0\end{array}$ & $\begin{array}{c}6 \\
12.0\end{array}$ & $\begin{array}{c}16 \\
32.0\end{array}$ & $\begin{array}{c}24 \\
48.0\end{array}$ & $\begin{array}{c}3 \\
6.0\end{array}$ & 3.44 & Sederhana \\
\hline 5. & $\begin{array}{l}\text { Rakan sebaya tidak membantu saya dalam } \\
\text { menguasai bahasa isyarat }\end{array}$ & $\begin{array}{c}3 \\
6.0\end{array}$ & $\begin{array}{c}7 \\
14.0\end{array}$ & $\begin{array}{c}21 \\
42.0\end{array}$ & $\begin{array}{c}16 \\
32.0\end{array}$ & $\begin{array}{c}3 \\
6.0\end{array}$ & 3.18 & Sederhana \\
\hline 6. & $\begin{array}{l}\text { Kekurangan sumber buku untuk rujukan } \\
\text { pembelajaran bahasa isyarat }\end{array}$ & $\begin{array}{c}2 \\
4.0\end{array}$ & $\begin{array}{c}5 \\
10.0\end{array}$ & $\begin{array}{c}11 \\
22.0\end{array}$ & $\begin{array}{c}28 \\
56.0\end{array}$ & $\begin{array}{c}4 \\
8.0\end{array}$ & 3.54 & Sederhana \\
\hline 7. & $\begin{array}{l}\text { Saya mempunyai kekangan dari segi masa } \\
\text { untuk belajar bahasa isyarat }\end{array}$ & $\begin{array}{c}5 \\
10.0\end{array}$ & $\begin{array}{c}8 \\
16.0\end{array}$ & $\begin{array}{c}22 \\
44.0\end{array}$ & $\begin{array}{c}14 \\
28.0\end{array}$ & $\begin{array}{c}1 \\
2.0\end{array}$ & 2.96 & Sederhana \\
\hline 8. & $\begin{array}{l}\text { Tahap penguasaan bahasa isyarat saya yang } \\
\text { lambat. }\end{array}$ & 0 & $\begin{array}{c}1 \\
2.0\end{array}$ & $\begin{array}{c}34 \\
68.0\end{array}$ & $\begin{array}{c}12 \\
24.0\end{array}$ & $\begin{array}{c}3 \\
6.0\end{array}$ & 3.34 & Sederhana \\
\hline 9. & Tidak minat untuk belajar bahasa isyarat. & $\begin{array}{c}2 \\
4.0\end{array}$ & $\begin{array}{c}27 \\
54.0\end{array}$ & $\begin{array}{c}12 \\
24.0\end{array}$ & $\begin{array}{c}8 \\
16.0\end{array}$ & $\begin{array}{c}1 \\
2.0\end{array}$ & 2.58 & Sederhana \\
\hline 10. & $\begin{array}{l}\text { Kekurangan laman web untuk pembelajaran } \\
\text { bahasa isyarat. }\end{array}$ & $\begin{array}{c}3 \\
6.0\end{array}$ & $\begin{array}{c}5 \\
10.0\end{array}$ & $\begin{array}{c}9 \\
18.0\end{array}$ & $\begin{array}{c}30 \\
60.0\end{array}$ & $\begin{array}{c}3 \\
6.0\end{array}$ & 3.50 & Sederhana \\
\hline 11. & $\begin{array}{l}\text { Jarang berkomunikasi menggunakan bahasa } \\
\text { isyarat }\end{array}$ & $\begin{array}{c}5 \\
10.0\end{array}$ & $\begin{array}{c}20 \\
40.0\end{array}$ & $\begin{array}{c}11 \\
22.0\end{array}$ & $\begin{array}{c}14 \\
28.0\end{array}$ & 0 & 2.68 & Sederhana \\
\hline 12. & $\begin{array}{l}\text { KTBM hanya digunakan semasa di dalam } \\
\text { bilik darjah sahaja. }\end{array}$ & $\begin{array}{c}1 \\
2.0\end{array}$ & $\begin{array}{c}13 \\
26.0\end{array}$ & $\begin{array}{c}17 \\
34.0\end{array}$ & $\begin{array}{c}13 \\
26.0\end{array}$ & $\begin{array}{c}6 \\
12.0\end{array}$ & 3.20 & Sederhana \\
\hline 13. & $\begin{array}{l}\text { Saya kerap menggunakan BIM semasa } \\
\text { berkomunikasi }\end{array}$ & $\begin{array}{c}2 \\
4.0\end{array}$ & $\begin{array}{c}5 \\
10.0\end{array}$ & $\begin{array}{c}28 \\
56.0\end{array}$ & $\begin{array}{c}11 \\
22.0\end{array}$ & $\begin{array}{c}4 \\
8.0\end{array}$ & 3.20 & Sederhana \\
\hline 14. & $\begin{array}{l}\text { Perkataan bahasa isyarat yang dibukukan } \\
\text { sangat terhad. }\end{array}$ & 0 & $\begin{array}{c}4 \\
8.0\end{array}$ & $\begin{array}{c}11 \\
22.0\end{array}$ & $\begin{array}{c}23 \\
46.0\end{array}$ & $\begin{array}{c}12 \\
24.0\end{array}$ & 3.86 & Tinggi \\
\hline 15. & $\begin{array}{l}\text { BIM lebih senang untuk dipelajari } \\
\text { berbanding KTBM }\end{array}$ & $\begin{array}{c}3 \\
6.0 \\
\end{array}$ & $\begin{array}{c}6 \\
12.0 \\
\end{array}$ & $\begin{array}{c}21 \\
42.0\end{array}$ & $\begin{array}{c}15 \\
30.0 \\
\end{array}$ & $\begin{array}{c}5 \\
10.0\end{array}$ & 3.26 & Sederhana \\
\hline & Jumlah purata min keseluruhan & & & & & & 3.16 & Sederhana \\
\hline
\end{tabular}

Table 8. Halangan dan Cabaran Guru Pendidikan Khas Bermasalah Pendengaran dalam Mempelajari Bahasa Isyarat

\begin{tabular}{|c|c|c|c|c|c|c|c|c|}
\hline BIL & PENYATAAN & $\begin{array}{c}\text { STS } \\
\text { f } \\
\%\end{array}$ & $\begin{array}{c}\text { TS } \\
\text { f } \\
\%\end{array}$ & $\begin{array}{c}\text { TP } \\
\mathbf{f} \\
\%\end{array}$ & $\begin{array}{c}\mathbf{S} \\
\mathbf{f} \\
\%\end{array}$ & $\begin{array}{c}\text { SS } \\
\text { f } \\
\%\end{array}$ & MIN & Markah \\
\hline 1. & $\begin{array}{l}\text { Saya sukar mempelajari bahasa isyarat } \\
\text { kerana kekurangan kemudahan ICT. }\end{array}$ & 0 & $\begin{array}{c}2 \\
20.0\end{array}$ & $\begin{array}{c}6 \\
60.0\end{array}$ & $\begin{array}{c}2 \\
20.0\end{array}$ & 0 & 3.00 & Sederhana \\
\hline 2. & $\begin{array}{l}\text { KTBM mengambil masa yang lama untuk } \\
\text { dipelajari dan dihafal }\end{array}$ & $\begin{array}{c}1 \\
10.0\end{array}$ & $\begin{array}{c}2 \\
20.0\end{array}$ & $\begin{array}{c}6 \\
60.0\end{array}$ & $\begin{array}{c}1 \\
10.0\end{array}$ & 0 & 2.70 & Sederhana \\
\hline 3. & Saya malas untuk belajar bahasa isyarat & $\begin{array}{c}2 \\
20.0\end{array}$ & $\begin{array}{c}6 \\
60.0\end{array}$ & $\begin{array}{c}2 \\
20.0\end{array}$ & 0 & 0 & 2.00 & Rendah \\
\hline 4. & $\begin{array}{l}\text { Saya mengalami kecelaruan dan kekeliruan } \\
\text { antara BIM dan KTBM. }\end{array}$ & $\begin{array}{c}1 \\
10.0\end{array}$ & $\begin{array}{c}1 \\
10.0\end{array}$ & $\begin{array}{c}3 \\
30.0\end{array}$ & $\begin{array}{c}5 \\
50.0\end{array}$ & 0 & 3.20 & Sederhana \\
\hline 5. & $\begin{array}{l}\text { Rakan sebaya tidak membantu saya dalam } \\
\text { menguasai bahasa isyarat }\end{array}$ & $\begin{array}{c}3 \\
30.0\end{array}$ & $\begin{array}{c}5 \\
50.0\end{array}$ & $\begin{array}{c}1 \\
10.0\end{array}$ & $\begin{array}{c}1 \\
10.0\end{array}$ & & 2.00 & Rendah \\
\hline 6. & $\begin{array}{l}\text { Kekurangan sumber buku untuk rujukan } \\
\text { pembelajaran bahasa isyarat }\end{array}$ & 0 & $\begin{array}{c}1 \\
10.0\end{array}$ & $\begin{array}{c}3 \\
30.0\end{array}$ & $\begin{array}{c}3 \\
30.0\end{array}$ & $\begin{array}{c}3 \\
30.0\end{array}$ & 3.80 & Tinggi \\
\hline
\end{tabular}




\begin{tabular}{|c|c|c|c|c|c|c|c|c|}
\hline 7. & $\begin{array}{l}\text { Saya mempunyai kekangan dari segi masa } \\
\text { untuk belajar bahasa isyarat }\end{array}$ & $\begin{array}{c}1 \\
10.0\end{array}$ & $\begin{array}{c}2 \\
20.0\end{array}$ & $\begin{array}{c}2 \\
20.0\end{array}$ & $\begin{array}{c}5 \\
50.0\end{array}$ & 0 & 3.10 & Sederhana \\
\hline 8. & $\begin{array}{l}\text { Tahap penguasaan bahasa isyarat saya yang } \\
\text { lambat. }\end{array}$ & $\begin{array}{c}1 \\
10.0\end{array}$ & $\begin{array}{c}2 \\
20.0\end{array}$ & $\begin{array}{c}5 \\
50.0\end{array}$ & $\begin{array}{c}2 \\
20.0\end{array}$ & 0 & 2.80 & Sederhana \\
\hline 9. & Tidak minat untuk belajar bahasa isyarat. & $\begin{array}{c}2 \\
20.0\end{array}$ & $\begin{array}{c}5 \\
50.0\end{array}$ & $\begin{array}{c}2 \\
20.0\end{array}$ & $\begin{array}{c}1 \\
10.0\end{array}$ & 0 & 2.20 & Rendah \\
\hline 10. & $\begin{array}{l}\text { Kekurangan laman web untuk pembelajaran } \\
\text { bahasa isyarat. }\end{array}$ & $\begin{array}{c}1 \\
10.0\end{array}$ & $\begin{array}{c}1 \\
10.0\end{array}$ & $\begin{array}{c}3 \\
30.0\end{array}$ & $\begin{array}{c}4 \\
40.0\end{array}$ & $\begin{array}{c}1 \\
10.0\end{array}$ & 3.30 & Sederhana \\
\hline 11. & $\begin{array}{l}\text { Jarang berkomunikasi menggunakan bahasa } \\
\text { isyarat }\end{array}$ & $\begin{array}{c}2 \\
20.0\end{array}$ & $\begin{array}{c}4 \\
40.0\end{array}$ & $\begin{array}{c}3 \\
30.0\end{array}$ & $\begin{array}{c}1 \\
10.0\end{array}$ & 0 & 2.30 & Rendah \\
\hline 12. & $\begin{array}{l}\text { KTBM hanya digunakan semasa waktu } \\
\text { pengajaran dan pembelajaran sahaja. }\end{array}$ & $\begin{array}{c}3 \\
30.0\end{array}$ & $\begin{array}{c}2 \\
20.0\end{array}$ & $\begin{array}{c}1 \\
10.0\end{array}$ & $\begin{array}{c}4 \\
40.0\end{array}$ & 0 & 2.60 & Sederhana \\
\hline 13. & $\begin{array}{l}\text { Saya kerap menggunakan BIM semasa } \\
\text { berkomunikasi }\end{array}$ & $\begin{array}{c}2 \\
20.0\end{array}$ & $\begin{array}{c}2 \\
20.0\end{array}$ & $\begin{array}{c}5 \\
50.0\end{array}$ & $\begin{array}{c}1 \\
10.0\end{array}$ & 0 & 2.50 & Sederhana \\
\hline 14. & $\begin{array}{l}\text { Perkataan bahasa isyarat yang dibukukan } \\
\text { sangat terhad. }\end{array}$ & $\begin{array}{c}1 \\
10.0\end{array}$ & $\begin{array}{c}1 \\
10.0\end{array}$ & $\begin{array}{c}5 \\
50.0\end{array}$ & $\begin{array}{c}1 \\
10.0\end{array}$ & $\begin{array}{c}2 \\
20.0\end{array}$ & 3.20 & Sederhana \\
\hline 15. & $\begin{array}{l}\text { BIM lebih senang untuk dipelajari } \\
\text { berbanding KTBM }\end{array}$ & $\begin{array}{c}1 \\
10.0\end{array}$ & $\begin{array}{c}1 \\
10.0\end{array}$ & $\begin{array}{c}6 \\
60.0\end{array}$ & $\begin{array}{c}2 \\
20.0\end{array}$ & 0 & 2.90 & Sederhana \\
\hline & Jumlah purata min keseluruhan & & & & & & 2.78 & Sederhana \\
\hline
\end{tabular}

\section{METHOD}

This study has three research objectives, as follows: (1) To identify the skill level of sign language for deaf students and special education teacher. (2) To identify the learning media of sign language for deaf students and special education teachers. (3) To identify the barriers and challenges faced by deaf students and special education teachers in learning sign language.

This study conducts a descriptive survey using questionnaires. The focus of the research is to see learning sign language for deaf students. The researcher also wants to study the obstacles and challenges faced by deaf students and special education teachers in learning sign language. Furthermore, this study also wants to determine the best method for teaching and learning sign language for deaf students and their teacher.

In this study, a total of 50 deaf students and 10 special education teacher of the deaf in Melaka Secondary High School are selected as sample. The population of this study is students from special education of the deaf in Melaka. Students are selected randomly and from different levels of age and gender.

Researchers use an adapted questionnaire for this study. Researchers use a modified questionnaire to obtain the appropriate data. A five-point Likert scale is used, as follow: (1) Strongly disagree (SD); (2) Disagree (D); (3) Not sure (NS); (4) Agree (A); (5) Strongly agree (SA)

The tool used in this study is the item questions which must be filled by deaf students and special education teachers to get demographic information, learning method, mastery level of sign language, challenges and obstacles faced during the process of learning sign language.

\section{FINDINGS AND DISCUSSION}

\section{Findings}

The results of the data analysis describe demographic distribution of the respondents, as in the table 1 until table 8 .

\section{Discussion}

The level of Sign Language Proficiency of Deaf Student and Special Education Teacher

Sign language is the communication language used by deaf students to interact. Sign Language combines form of hand signals, gestures, arm, body, and facial expressions to convey a message or to create a communication process. According to Wilbur (2013) sign language can also be used by communities that have problems in speaking and communities that are not able to listen.

In General, this research has found that level of Sign Language proficiency for deaf students is at moderate level. It is seen through the mean value of the highest level of mastery of sign language to help their language development. This is supported by Ibrahim (2000) which states that the effect KTBM is a major factor affecting deaf student performance in writing their Malay language.

The finding also shows that BIM is not a major communications media used by deaf students. BIM is not used in the process of teaching and learning at school because BIM does not have the appropriate structure and grammar to meet malay language stucture. BIM is only conducted in their communities. It is an informal communication media (Wei, 2007). 
In addition, the finding about the level of proficiency of teacher is also at a moderate level. This is shown by the findings of research in which most teachers support that level of Sign Language proficiency is very helpful to language development. It is supported by Yasin (1999) which states that deaf students who adopted the method of KTBM are fully able to demonstrate a high level of achievement in test than students from the merger school that is provided less adept at using KTBM method.

The findings about a special education teacher of the deaf who recorded the lowest mean score shows that most respondents agree that BIM is not the major sign language used by them. This is because the system used in BIM is not the same with Malay language. BIM is a language that is built using its own grammatical structure of the deaf community. Sign language is a language that is practiced through a sign method manually and is applied using eyes to receive and using hand and facial expression to express. BIM uses a special syntax structures such as verb + object in sentences such as "eating the fruit" which means consuming fruits which does not meet the features of correct grammar of Malay language.

BIM is not recommended in use at the school because the sentence structure does not meet the proper Malay language. This will hamper language development of students and affect their academic performance. It is also supported by Wei (2004) which states BIM has its unique characteristic and does not conform to the policy of the Malay language in the Malay language principle.

The Media of Learning Sign Language Used by Deaf Student and Special Education Teacher of The Deaf

Analysis of the research findings toward learning media of sign language used by the deaf students show a mean value at a medium level. Based on the highest mean value, most respondents agree that the learning media of sign language through books are more easily understood. This shows that most students learn sign language through books. The book is a hard copy material that is easy to find and to use. According to Fang (2007) BKTM introduced in 1978 has shown that 1612 sign language codes have been successfully produced. This signal code has been printed in three volumes with the title communication: sign language. With the provision of books for reference would make it easier language learning process for students because they can master new words gradually. This method can also help the process of communication and interaction.

The lowest mean value shows that deaf students do not agree with items that they learn sign language through the website. This item is rejected because sign language learning resources through the web are very limited. According to Gray (1995) in berhmann who has conducted a survey on educational software for deaf students in 1984 did not find a lot of software designed for people with hearing barrier. Therefore, the resources to access the web site for learning sign language is less and difficult because the source is not friendly and not variative. This causes the majority of students do not give more emphasis on learning through the website.

Analysis of the overall mean value of media learning sign language of teacher in special education also shows a high level. The highest mean values show that most respondents agreed that media learning through communication and interaction with coworkers can help mastering their sign language. Visual interaction will directly give high understanding of the individual. It is also supported by Yusof (1995) which states that a real thing for the hearing problem is the inclusion of information through vision. Eyesight is able to receive information more quickly and be easily accessible. Vision is able to detect and capture clear physical symbol without ambiguity for a receiver and a transmitter. This proves that visual learning is stimulating the development of the mind of an individual, especially the hearing. According Redziewicz and Antonellis (1993) states that the deaf people relied vision much to get information in their minds. They are said to be able to master the concrete than abstract. Thus, the frequency of interaction with colleagues and students can accelerate the process of learning of sign language teachers. This is because, the existence of obligation for the teachers to speed up the sign language skills

The lowest mean value indicates that most special education teachers of the deaf do not agree with the item stating that they learn sign language through the website. This is because the source of learning sign language through the website is very less. This is due to the lack of skilled teachers in the field of ICT. Some teachers that are less skilled in the use of ICT resources cause them less access websites for learning purposes. This statement is supported by Chu Shi Harn (2005) special education teachers in Melaka have a less degree of readiness to use computers in teaching and learning process. In addition, Noraini binti Attan (2002) states that the limited time faced by most teachers cause them do not have enough time to study the field of information technology. This is because the burden of administration tasks that must be done by the teachers make them less enough time to use the website.

Barriers and Challenges Faced by Deaf Student and Special Education Teacher of The Deaf in Learning Sign Language

The research findings of the obstacles and 
challenges faced by deaf students in learning sign language show the mean value at a modest rate. It is generally understood that sign language is the main language for deaf students. They have to master sign language to interact with the surrounding society as well as their communities. Barriers in terms of the lack of sign language symbol that is booked become the main stumbling factor for deaf students. Lack of resources such as book reference cause them do not have a reference source. This can be shown by the total lack of KTBM's words booked compare to Malay dictionary containing about 5834 basic words (dictionary Council, 2005 ) and only about $0.08 \%$ of the amount produced by KTBM (KTBM volume 1 and 2).

In addition, the statements regarding barriers of laziness are not supported by student impaired and has recorded the lowest mean value. This is because sign language is a language that must be used by them to interact. This has been explained by Yusof (1994), KTBM a standard language for the deaf. KTBM not only their mother language but the language should be studied and explored in order to improve their communication. In terms of grammar and semantics, KTBM clearly shows that it is entirely based on the Malay language.

The analysis of overall mean value for special education teacher of the deaf is also at a modest rate. The highest mean value for the barriers faced by teachers to learn sign language is the lack of resources in respect of the items used as reference books. Most respondents agree with this item. In 1985, in collaboration with the Ministry of Education Malaysia and language and reference council, BMKT code has been renovated and upgraded. This code has been recorded and published as the book entitled "The Malay Communications Hands code volume 1 (Ministry of Education Malaysia, 1985). However, constraints in terms of its cooperation with certain departments do not plan smoothly. This situation causes the process to publish the book down. As it is known, sign language book which was published is only a few volumes and without adding a new word. This is proven by the lack of a significant number of words of KTBM (KTBM volumes 1 and 2) compared to the number of words in the Malay dictionary (Kamus Dewan, 2005).

Furthermore, the items that recorded the lowest mean value have indicated that most respondents disagreed with the statement inhibition of peers who did not help. This statement is rejected because a lot of colleagues and students help learning a language by teacher. Guidance and direct interaction with individual can improve the brain's ability to record and save something. This is supported by the statement of Yusof (1994) that because BMKT or KTBM is a new language, the one who uses it is limited. In the use of this KTBM they are encouraged to sound voice like talking with the other because for individuals who have residual hearing can train the senses of hearing to hear a speech and language as well as can introduce noise to them. Thus, direct interaction can stimulate the sense of hearing and can make it easier for respondents to learn sign language.

\section{CONCLUSION AND SUGGESTION}

This study aims to identify how to learn sign anguage by deaf students and the level of mastery of sign language by students and teacher of the deaf at a state school in Melaka. In addition, this research would also like to know the major obstacles faced by deaf students and teachers who learn sign language. The findings indicate that the level of mastery of sign language for problematic pupils hearing and special education teachers is at mid-level. They completely use Kode Tangan Bahasa Melayu in their daily teaching and communication. The way to learn sign language for deaf student is through the book while special education teachers also learn it through colleagues. The challenges as well as the obstacles often encountered to learn sign language for deaf students and special education teachers of the deaf are lack of source reference book of sign language. It is suggested that reference materials such as book and ICT are augmented in all integration program and special education of the deaf.

\section{REFERENCE}

Fang, Y. S. (2007). Pengaruh BIM Penggunaan KTBM di Kalangan Pelajar Masalah Pendengaran. Universiti Kebangsaan Malaysia.

Goh, O. S., \& Teh, K. H. (1993). Bahasa tulisan pelajar pekak: analisis struktur sintaksis ayat berdasarkan teori tatabahasa transformasi generatif. Dewan Bahasa dan Pustaka, Kementerian Pendidikan Malaysia.

Ibrahim, R. (2000). Pencapaian Pelajar Cacat Pendengaran Dalam Penulisan Karangan Bahasa Melayu. Universiti Kebangsaan Malaysia.

Paul, P. \& Quigley, S. (1994). Language And Deafness. San Diego: Singular Publishing Group.

Safani, B. (2012). Kaedah Pengajian Kontekstual Bahasa Melayu dalam Kalangan Murid-murid Bermasalah Pendengaran. Universiti Kebangsaan Malaysia.

Wei, H. K. (2004). Syntax in Malaysian Sign Language. Master in Lingustic. Gallaudet University.

Wei, H. K. (2007). Bahasa Isyarat Malaysia: Satu Pendekatan Linguistik. Seminar Kebangsaan Bahasa Verbal dan Bukan Verbal dari Perspektif Bahasa dan Terjemahan Komunikasi. Pulau Pinang. Universiti Sains Malaysia. 
Yasin, M. H. M. (1999). Bahasa Malaysia Kod Tangan (BMKT) Penggunaan dalam Pengajaran kepada Pelajar-pelajar Pekak di Sekolah Menengah di Negeri Melaka. Universiti Kebangsaan Malaysia.

Yasin, M. H. M. (2005). Perbandingan Pencapaian Pelajar Pendidikan Khas (Pekak) Menggunakan Tiga Format Peperiksaan Ujian Penilaian Sekolah Rendah (UPSR). Tesis Doktor Falsafah, Fakulti Pendidikan, Universiti Kebangsaan Malaysia : Bangi.

Yusof, A. (1994). Komunikasi Seluruh Bahasa Malaysia Kod Tangan (BMKT): Bahasa Komunikasi Pelajar Pekak. Jurnal Dewan Bahasa.

Yusof, A. (1995). Fikiran, Persekitaran dan Pembentukan Isyarat Orang Pekak. Jurnal Dewan bahasa. 